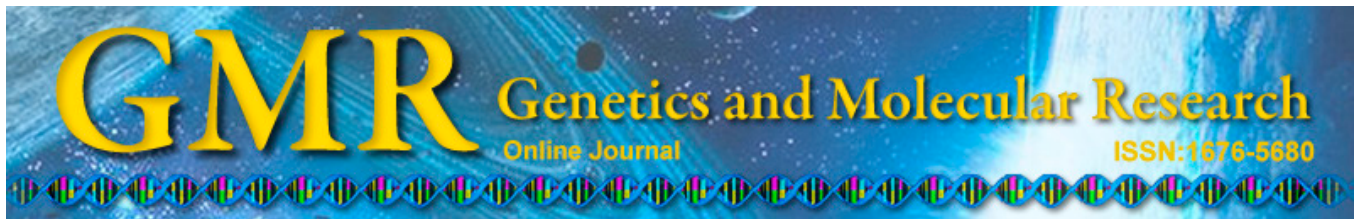

\title{
A novel random amplified polymorphic DNA- based strategy for genetic diversity analysis and identification of tomatoes
}

\author{
X. Cao, Z. Wu, R. Zhou, F.L. Jiang and Z.E. Yang \\ Key Laboratory of Horticultural Plant Biology and Germplasm Innovation \\ in East China, Ministry of Agriculture, College of Horticulture, \\ Nanjing Agricultural University, Nanjing, China \\ Corresponding author: $\mathrm{Z}$. Wu \\ E-mail:wzh@njau.edu.cn
}

Genet. Mol. Res. 14 (1): 1650-1661 (2015)

Received February 10, 2014

Accepted June 10, 2014

Published March 6, 2015

DOI http://dx.doi.org/10.4238/2015.March.6.11

\begin{abstract}
Cultivar identification diagrams (CIDs) provide a rapid and efficient approach for identifying cultivars based on random amplified polymorphic DNA (RAPD) markers. In this paper, 64 tomato cultivars were identified using a CID. Using RAPD profiles, clustering analysis was performed to analyze genetic diversity. The results showed that 8 RAPD primers could completely separate the 64 cultivars according to the obtained polymorphic bands; a CID of the 64 tomato cultivars was then constructed. As verification of the CID validity, 8 randomly selected cultivars were investigated and proven to be well distinguished. In addition, 33 DNA bands were obtained, 20 (60.6\%) of which were polymorphic. Genetic distances were calculated with a range of 0.032 to 1.402 . Clustering analysis showed that the 64 tomato cultivars were divided into 4 groups with a similarity coefficient of 0.40 . Using this novel strategy, with the same RAPD data, both CID and clustering analysis can simultaneously determine tomato cultivars and their genetic diversity.
\end{abstract}

Key words: Tomato; Random amplified polymorphic DNA; Cultivar identification diagram (CID); Cultivar identification; Genetic diversity analysis 


\section{INTRODUCTION}

The tomato, the annual or perennial herbage of Lycopersicon of Solanaceae, originates from Peru and Ecuador in South America and is cultivated worldwide. With extensive adaptability, tomato plants can grow from $45^{\circ}$ south latitude to $65^{\circ}$ north latitude. Germplasm resources of tomatoes are plentiful, including one cultivated species and 12 wild relatives (Rick et al., 1990; Peralta et al., 2006). Following the introduction, breeding, and domestication of the tomato, more and more cultivars have been developed. Subsequently, the cultivar chaos phenomenon became an issue, which brings with it difficulties for cultivar application and research. Therefore, establishing an effective strategy to distinguish tomato cultivars is essential.

Traditional approaches for tomato cultivar identification are usually based on morphological and physiological characteristics; however, this procedure is easily influenced by the surrounding environment and is a time-consuming practice (Baird et al., 1996). In addition, when considering the differentiation of closely related species, this can be an extremely difficult work. Therefore, to establish an effective strategy to identify tomato germplasms is very important. Furthermore, the tomato has become an excellent model system for studying genetic engineering, plant tissue culture, and molecular genetics. The identification of tomato cultivars provides a basis for tomato germplasm and molecular biology research, which have great theoretical significance and practical value.

Molecular markers are powerful tools for assessing genetic diversity and identifying cultivars. Many DNA-based marker systems, such as random amplified polymorphic DNA (RAPD), simple sequence repeat, amplified fragment length polymorphism, sequence characterized amplified region, and sequence-related amplified polymorphism have been used in this discipline (Palombi and Damiano, 2002; Lee et al., 2004; Fang et al., 2006; Comlekcioglu et al., 2010; Aruga et al., 2012). Among these, RAPD has proven to be a particularly useful means for cultivar analysis and genetic diversity detection (Williams et al., 1990) because it has the advantage of speed and simplicity. However, to our knowledge, traditional analytic methods, such as clustering analysis, have many deficiencies because they are based on many different primer designs, and the output result is only in a digital form generated by software calculations. In other words, the traditional method requires much work and cannot provide polymorphic primers and markers to distinguish one cultivar from another.

Cultivar identification diagrams (CIDs) by RAPD were developed in the last 3years, and it has so far been used for the identification of sweet orange, pear, grapevine, loquat, and apple cultivars by Fang's group (Lin et al., 2011; Zhang et al., 2011; Zhao et al., 2011; Sun et al., 2012; Wang et al., 2012). As a novel strategy for cultivar identification using RAPD markers, CID overcomes the defects of the traditional analytic method, requiring fewer primers for RAPD-polymerase chain reaction (PCR) amplification, and the results are output in diagram form. However, the CID strategy cannot be used to efficiently analyze the genetic diversity of plant cultivars.

In order to overcome the deficiencies of existing methods, we used the same RAPD profiles for clustering analysis and CID by RAPD to simultaneously identify tomato cultivars and their genetic diversity. This novel strategy provides a more rapid, powerful, and efficient means of identifying tomato cultivars and their genetic diversity than previously reported. 


\section{MATERIAL AND METHODS}

\section{Plant material}

A total of 64 cultivars were used in this study (Table 1). Of the 64 cultivars, 58 were introduced from the Tomato Genetic Resource Center, University of California, and 6 tomato cultivars were from the People's Republic of China; all 64 tomato cultivars were assessed with RAPD markers (Table 2).

Table 1. Sources of tomato cultivars used in this experiment.

\begin{tabular}{|c|c|c|c|c|c|c|c|}
\hline No. & Accessions & Species & Origin $^{\mathrm{a}}$ & No. & Accessions & Species & Origin $^{a}$ \\
\hline 1 & LA4031 & S. lycopersicum & TGRC & 33 & LA0490 & S. lycopersicum & TGRC \\
\hline 2 & LA4032 & S. lycopersicum & TGRC & 34 & LA 1222 & S. lycopersicum & TGRC \\
\hline 3 & LA4037 & S. lycopersicum & TGRC & 35 & LA1598 & S. pimpinellifolium & TGRC \\
\hline 4 & LA4038 & S. lycopersicum & TGRC & 36 & LA1627 & S. galapagense & TGRC \\
\hline 5 & LA4040 & S. lycopersicum & TGRC & 37 & LA 1698 & S. lycopersicum & TGRC \\
\hline 6 & LA4043 & S. lycopersicum & TGRC & 38 & LA 1777 & S. habrochaites & TGRC \\
\hline 7 & LA4044 & S. lycopersicum & TGRC & 39 & LA2093 & S. pimpinellifolium & TGRC \\
\hline 8 & LA4047 & S. lycopersicum & TGRC & 40 & LA2413 & S. lycopersicum & TGRC \\
\hline 9 & LA4054 & S. lycopersicum & TGRC & 41 & LA2661 & S. lycopersicum & TGRC \\
\hline 10 & LA4055 & S. lycopersicum & TGRC & 42 & LA2662 & S. lycopersicum & TGRC \\
\hline 11 & LA4063 & S. lycopersicum & TGRC & 43 & LA2683 & S. lycopersicum & TGRC \\
\hline 12 & LA4064 & S. lycopersicum & TGRC & 44 & LA2706 & S. lycopersicum & TGRC \\
\hline 13 & LA4065 & S. lycopersicum & TGRC & 45 & LA2838A & S. lycopersicum & TGRC \\
\hline 14 & LA4071 & S. lycopersicum & TGRC & 46 & LA 3120 & S. lycopersicum & TGRC \\
\hline 15 & LA4081 & S. lycopersicum & TGRC & 47 & LA3183 & S. lycopersicum & TGRC \\
\hline 16 & LA4089 & S. lycopersicum & TGRC & 48 & LA3320 & S. lycopersicum & TGRC \\
\hline 17 & LA4091 & S. lycopersicum & TGRC & 49 & LA3473 & S. lycopersicum & TGRC \\
\hline 18 & LA4099 & S. lycopersicum & TGRC & 50 & LA3475 & S. lycopersicum & TGRC \\
\hline 19 & LA4030 & S. lycopersicum & TGRC & 51 & LA3538 & S. lycopersicum & TGRC \\
\hline 20 & LA4034 & S. lycopersicum & TGRC & 52 & LA3847 & S. lycopersicum & TGRC \\
\hline 21 & LA4036 & S. lycopersicum & TGRC & 53 & LA3915 & S. lycopersicum & TGRC \\
\hline 22 & LA4042 & S. lycopersicum & TGRC & 54 & LA3916 & S. lycopersicum & TGRC \\
\hline 23 & LA4068 & S. lycopersicum & TGRC & 55 & LA3918 & S. lycopersicum & TGRC \\
\hline 24 & LA4072 & S. lycopersicum & TGRC & 56 & LA3919 & S. lycopersicum & TGRC \\
\hline 25 & LA4073 & S. lycopersicum & TGRC & 57 & LA3920 & S. lycopersicum & TGRC \\
\hline 26 & LA4082 & S. lycopersicum & TGRC & 58 & LA4355 & S. lycopersicum & TGRC \\
\hline 27 & LA4083 & S. lycopersicum & TGRC & 59 & Zhongza No. 9 & S. lycopersicum & $\mathrm{CH}-\mathrm{BJ}$ \\
\hline 28 & LA4090 & S. lycopersicum & TGRC & 60 & Zhongza No. 105 & S. lycopersicum & $\mathrm{CH}-\mathrm{BJ}$ \\
\hline 29 & LA4096 & S. lycopersicum & TGRC & 61 & Zhongza No. 109 & S. lycopersicum & $\mathrm{CH}-\mathrm{BJ}$ \\
\hline 30 & LA4098 & S. lycopersicum & TGRC & 62 & Jiangshu No. 14 & S. lycopersicum & CH-JS \\
\hline 31 & LA4070 & S. lycopersicum & TGRC & 63 & Hezuo 908 & S. lycopersicum & $\mathrm{CH}-\mathrm{SH}$ \\
\hline 32 & LA0483 & S. galapagense & TGRC & 64 & Suhong 2003 & S. lycopersicum & CH-JS \\
\hline
\end{tabular}

aTGRC, courtesy of the Tomato Genetic Resource Center, University of California; CH-BJ, Beijing, China; CH-JS, Jiangsu, China; $\mathrm{CH}-\mathrm{SH}$, Shanghai, China.

Table 2. RAPD primers and annealing temperatures used in this experiment.

\begin{tabular}{llr}
\hline Primer & Nucleotide sequence $\left(5^{\prime} \rightarrow 3^{\prime}\right)$ & Annealing temperature $\left({ }^{\circ} \mathrm{C}\right)$ \\
\hline Y4 & GTTTCGCTCCT & 44.4 \\
Y22 & GGACCCAACCT & 42.8 \\
Y27 & GTGTGCCCCAA & 40.4 \\
Y28 & GTGTGCCCCAT & 43.7 \\
Y33 & AAGCCTCGTCA & 42.8 \\
Y39 & AGCGTCCTCCA & 42.6 \\
Y42 & AGCGTCCTCCC & 41.7 \\
Y53 & TGGTGGCGTTG & 43.7 \\
\hline
\end{tabular}




\section{Genomic DNA isolation}

Genomic DNA was isolated from the young leaves of 64 tomato seedlings using the modified cetyl trimethyl ammonium bromide method (Murray and Thompson, 1980; Bousquet et al., 1990). The quality of isolated genomic DNA was checked using a nucleic acid protein detector and then diluted to a final concentration of $30 \mathrm{ng} / \mu \mathrm{L}$ with $1 \mathrm{X}$ Tris base and ethylenediaminetetraacetic acid buffer and stored at $-20^{\circ} \mathrm{C}$ until use.

\section{RAPD-PCR analysis}

First, 60 RAPD 11-mer primers (Yu et al., 2009) were synthesized by Dingguo Changsheng Biotechnology company in Beijing, China. After PCR amplification, only primers with clear bands were selected for the next step. Based on this principle, 8 primers (Table 2) that showed clear and reproducible bands were utilized in cultivar identification. Amplification reactions were carried out in $20-\mu \mathrm{L}$ volumes, containing $2.0 \mu \mathrm{L} 10 \mathrm{X}$ buffer, $1.6 \mu \mathrm{L} 2.5 \mathrm{mM}$ dNTPs, $1.2 \mu \mathrm{L} 25 \mathrm{mM} \mathrm{MgCl}_{2}, 1.6 \mu \mathrm{L} 1.0 \mu \mathrm{M}$ primer, $0.08 \mu \mathrm{L} 5 \mathrm{U} / \mu \mathrm{L}$ rTaq polymerase Dynazyme, and $30 \mathrm{ng}$ genomic DNA. Amplification was performed in an Autorisierter Thermocycler (Eppendorf, Hamburg, Germany) using the following settings: initial denaturation at $94^{\circ} \mathrm{C}$ for $5 \mathrm{~min} ; 42$ cycles of $2 \mathrm{~min}$ for denaturation at $94^{\circ} \mathrm{C}, 1 \mathrm{~min}$ at annealing temperature (Table 2), and a 1 -min extension at $72^{\circ} \mathrm{C}$; and a final extension for 5 min at $72^{\circ} \mathrm{C}$. The PCR amplification products were isolated on $1.5 \%$ agarose gels electrophoretically at $100 \mathrm{~V}$ for 20 min, using $1 \mathrm{X}$ Tris base, acetic acid, and ethylenediaminetetraacetic acid buffer. Then, the gels were stained with $0.5 \mathrm{mg} / \mathrm{mL}$ ethidium bromide and photographed under ultraviolet light. DL 2000 plus marker was loaded and served as a standard molecular weight marker. All amplifications were repeated 3 times.

\section{Utilization and verification of the tomato CID}

Only the clear and specific bands photographed on the gel prints were selected for cultivar identification. For amplification by 1 primer, when some cultivars had 1 identically sized specific band in the fingerprint, they could be separated singly, while other cultivars sharing the same banding pattern were assigned to the same subgroup. More primers were used for identification until all cultivars could be gradually and completely distinguished by amplifying more specific bands. Based on this, a tomato CID was constructed according to the specific sizes of bands and their related primers.

Eight tomato cultivars that were randomly chosen from 2 different groups were used to verify the feasibility of the CID. If the randomly chosen cultivars could be distinguished accurately and quickly as shown in the complete CID, we would conclude that the method used in this research is a feasible way of identifying tomato cultivars.

\section{Genetic diversity analysis}

Polymorphic fragments amplified by primers used in 64 tomato cultivars were scored using a binary matrix: 1 for their presence and 0 for their absence. Genetic distances were calculated using the NTSYS-PC software version 2.11 (Rohlf, 2000). Clustering analysis was performed using the unweighted pair group method with arithmetic mean (UPGMA) and the 
TREECON program for Windows (version 1.36) neighbor joining and sequential agglomerative hierarchical and nested clustering.

\section{RESULTS}

\section{Characterization of RAPD-PCR-amplified products}

In order to create a suitable RAPD system with high reproducibility, 11-mer RAPD primers were initially utilized. If the primer that was randomly screened from a stock of 6011 mer primers could amplify clear fingerprints with polymorphic bands in 64 tomato cultivars, it was selected and further used for tomato cultivar identification. Through screening, 8 primers (Table 2) with high reproducibility and high resolution were obtained, which could completely distinguish all tomato cultivars. The number of derived bands and the polymorphisms revealed by each primer are shown in Table 3 . Considering all the primers and amplification products, a total of 33 bands were amplified by 8 primers, 20 of which were polymorphic with an average of 2.5 specific bands per primer. The percentage of polymorphism obtained from the 8 primers was different between cultivars; the maximum ratio was $100 \%$ produced by primer Y 53 , while the minimum value was $50 \%$ from primers Y4, Y28, and Y39. On average, the polymorphism percentage of the 8 primers reached $60.6 \%$.

Table 3. Diversity detected by RAPD primers.
\begin{tabular}{lccc}
\hline Primer & Total number of bands & Number of polymorphic bands & Percentage of polymorphism (\%) \\
\hline Y4 & 4 & 2 & 50.0 \\
Y22 & 5 & 3 & 60.0 \\
Y27 & 5 & 3 & 60.0 \\
Y28 & 6 & 3 & 50.0 \\
Y33 & 3 & 2 & 66.7 \\
Y39 & 4 & 2 & 50.0 \\
Y42 & 3 & 2 & 66.7 \\
Y53 & 3 & 3 & 100.0 \\
Total & 33 & 20 & - \\
Average/primer & 4.1 & 2.5 & 60.6 \\
\hline
\end{tabular}

\section{Cultivar identification by the CID method based on RAPD}

Based on the RAPD-PCR amplification products, 8 primers could distinguish all 64 tomato cultivars. Of these 8 primers, primer Y42 was initially used to detect DNA polymorphisms within all tomato cultivars; the electrophoresis gel image is shown in Figure 1. Primer Y42 generated clear and reproducible band patterns with 2 specific RAPD-PCR bands of 2800 and $1200 \mathrm{bp}$ in all 64 tomato cultivars. Using primer Y42, 64 tomato cultivars were divided into 3 groups: in group 1, the only cultivar (LA4072) with the presence of the 2800-bp band and the absence of the 1200-bp band was distinguished; group 2 consisted of 28 tomato cultivars including LA4031, LA4032, LA4038, LA4043, LA4081, LA4089, LA4073, LA4082, LA4083, LA4090, LA4096, LA4098, LA4070, LA0483, LA0490, LA1222, LA1627, LA1698, LA2093, LA2661, LA2662, LA2683, LA2706, LA2838A, LA3120, LA3183, LA3320, and Jiangshu No. 14, which were differentiated from others by the presence of both the 2800and 1200-bp bands; and the remaining 35 cultivars in group 3 included LA4037, LA4040, 
LA4044, LA4047, LA4054, LA4055, LA4063, LA4064, LA4065, LA4071, LA4091, LA4099, LA4030, LA4034, LA4036, LA4042, LA4068, LA1598, LA1777, LA2413, LA3473, LA3475, LA3538, LA3847, LA3915, LA3916, LA3918, LA3919, LA3920, LA4355, Zhongza No. 9, Zhongza No. 105, Zhongza No. 109, Hezuo 908, and Suhong 2003, which were characterized by the presence of the 1200-bp band and the absence of the 2800-bp band.

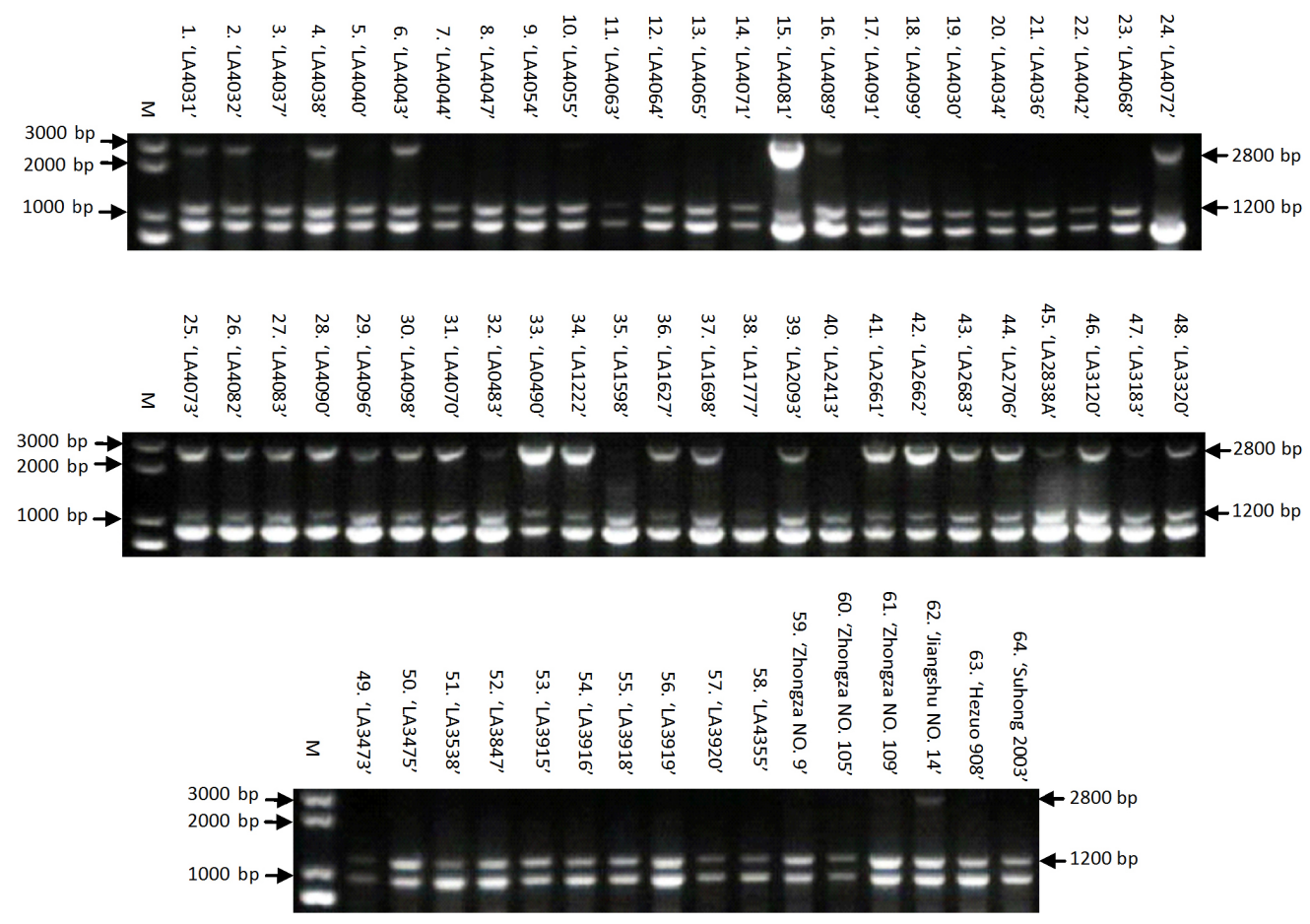

Figure 1. RAPD profile of 64 tomato cultivars obtained with primer Y42. Lanes and accessions of the cultivars are marked at the top. Lane $M=$ DL 2000 plus DNA marker.

Following this method, the other 7 primers and polymorphic bands obtained using them were screened step by step and chosen to identify the tomato cultivars until all cultivars could be completely separated as shown in Figure 2. For example, after the identification by Y42, primer Y4 was used to further separate cultivars from the previously obtained 3 groups. Group 1 included only 1 tomato cultivar; therefore, it was first separated from the others. Group 2 , which included 28 tomato cultivars, could be further divided into 4 subgroups using primer Y4; cultivars in subgroup 1, containing LA4032, LA4081, LA4073, LA2093, and Jiangshu No. 14, were identified by Y33, and LA2093 was distinguished from the other 4 cultivars by the presence of an 1100-bp band. Next, using primer Y53, LA4032 did not produce a 1300-bp band and was subsequently separated from the other 3 cultivars. For the remaining cultivars, LA4081 was identified using primer Y28 as it did not express an 1100-bp band, whereas LA4073 and Jiangshu No. 14 were finally differentiated by expression of an 1800-bp band.

Eventually, a tomato CID was constructed using the 8 polymorphic primers, which generated clear and specific sizes of bands using the stepwise identification method described 
above (Figure 2); therefore, this can be regarded as an effective and reliable tool for practical tomato cultivar identification.

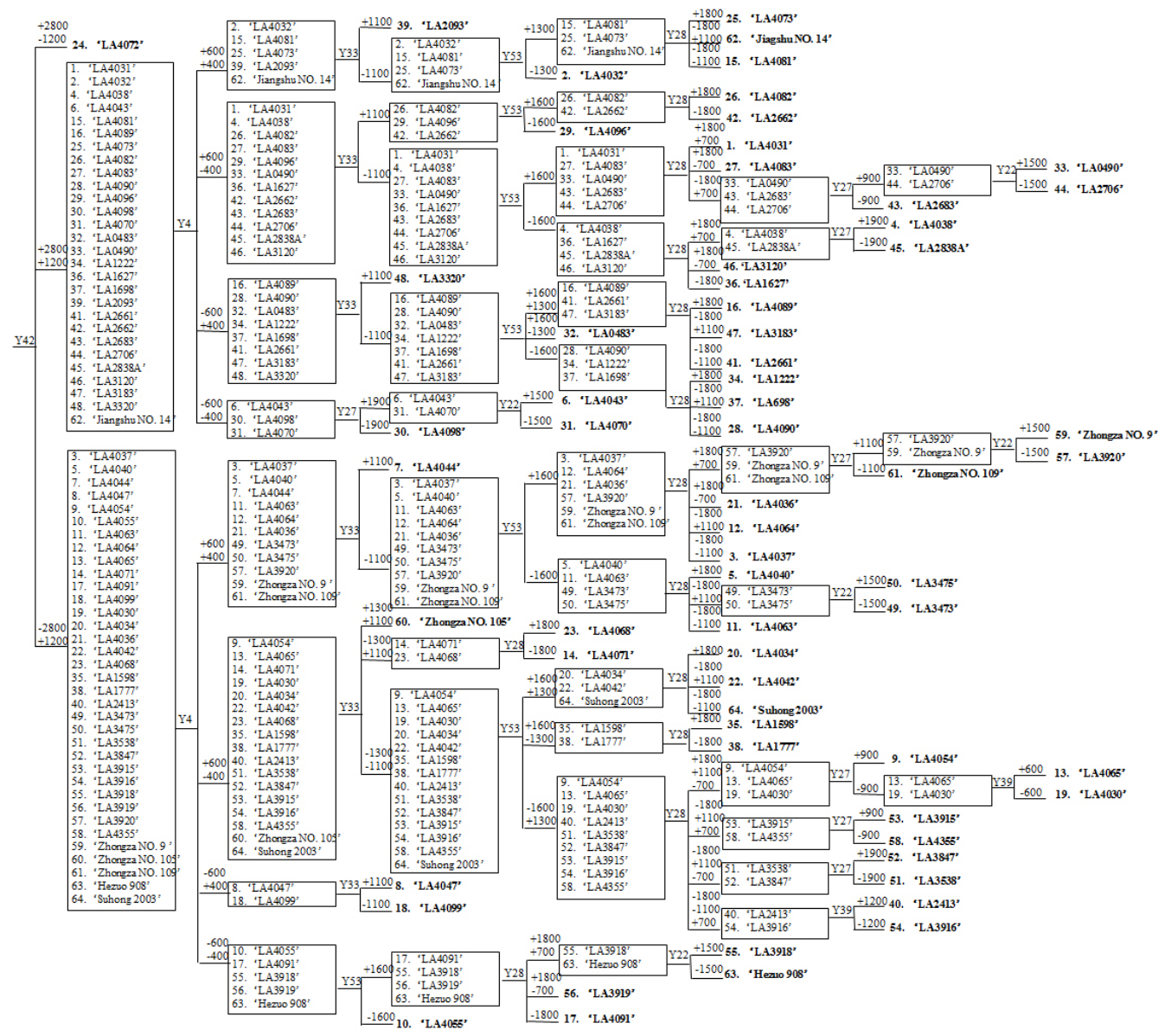

Figure 2. Cultivar identification diagram of tomato cultivars using eight primers. The lane number in the figure represents the size of the band; units are bp. $(+)$ indicates presence of the band; (-) indicates absence of the band. The cultivar names in boldface indicate those that were separated.

\section{Utilization and verification of the CID method based on RAPD}

To verify the accuracy and reproducibility of the CID method, 8 of the 64 tomato cultivars were randomly selected. First, 4 cultivars from group 1 (LA4037, LA4038, LA4082, and LA2838A) were tested. From the location of the 4 cultivars in the CID, it was easy to recognize that primers Y42, Y33, and Y27 could well distinguish the 4 cultivars from each other. Electrophoresis images showed that LA4037 was initially separated out from the other 3 cultivars using primer Y42 according to a 2800-bp RAPD-PCR band (Figure 3A). The 3 remaining cultivars were further differentiated by primer Y33, which generated an 1100-bp 
band, and LA4082 was separated out (Figure 3B). Lastly, LA4038 and LA2838A were divided by primer Y27 with a specific band of $1900 \mathrm{bp}$ (Figure 3C). In terms of the CID, the other 4 cultivars in group 2 (LA4065, LA4030, LA2413, and Suhong 2003) could be gradually distinguished using primers Y53, Y28, and Y39. In a manner similar to that described above, LA4065, LA4030, LA2413, and Suhong 2003 were effectively separated by the specific 1600bp (Figure 3D), 700-bp (Figure 3E), and 600-bp (Figure 3F) bands.

The results clearly show that the 8 randomly selected cultivars were verified in excellent agreement with the CID. Following this method, all 64 cultivars could be accurately identified using the CID and 8 polymorphic primers and their product-specific bands, which proved the suitability and efficiency of the CID method for tomato cultivar identification.
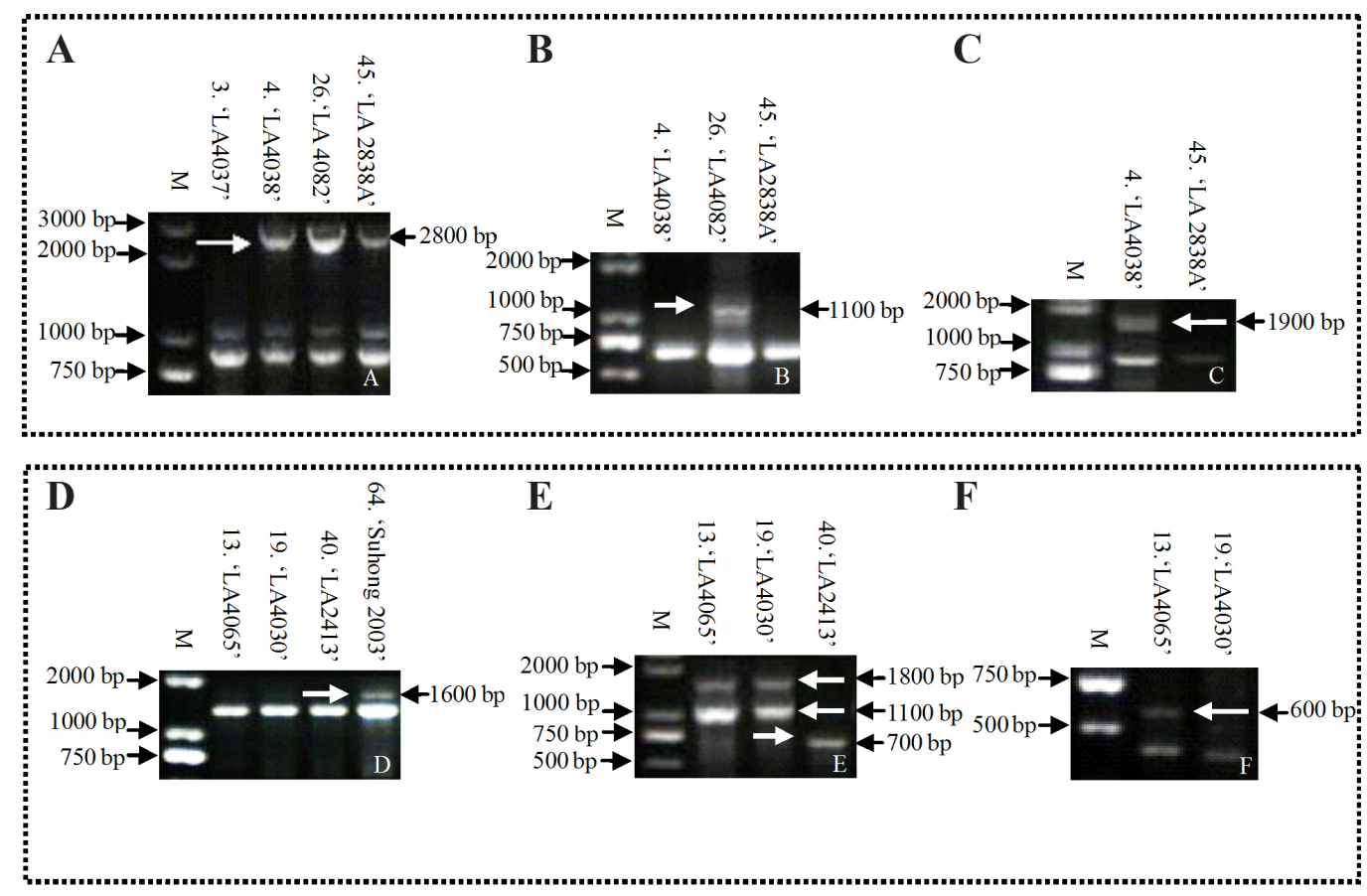

Figure 3. RAPD profiles for randomly selected tomato cultivars by corresponding primers. Lane $M=$ DL2000 plus marker. Group 1 includes A, B, and C. A. DNA banding patterns obtained with primer Y42. B. DNA banding patterns obtained with primer Y33. C. DNA banding patterns obtained with primer Y27. Group 2 includes D, E, and F. D. DNA banding patterns obtained with primer Y53. E. DNA banding patterns obtained with primer Y28. F. DNA banding patterns obtained with primer Y39. The specific bands are indicated by white arrows.

\section{Diversity analysis using clustering analysis based on RAPD}

To detect the genetic diversity among tomato cultivars and further verify the accuracy of the CID method described herein, clustering analysis was also established and used. Twenty polymorphic fragments from the 8 primers were scored as follows: 1 for presence or 0 for absence. The obtained data were used to calculate genetic distances using the NTSYS-pc program (Rohlf, 2000; version 2.11). The results indicated that genetic distances between the 64 
tomato cultivars ranged from 0.032 to 1.402 , with the highest and the lowest values of 1.402 between LA4099 and LA1777 and 0.032 between LA3920 and Zhongza No. 9, respectively. The large genetic distance difference that existed between cultivars reflects the wide range of genetic variations in the 64 tomato cultivars.

A dendrogram based on UPGMA analysis using RAPD markers was constructed (Figure 4), showing that the 64 tomato cultivars formed 4 distinct clusters with a similarity coefficient of 0.40 . Cluster I was the largest group and consisted of 50 tomato cultivars, while Cluster II contained 11 cultivars, Cluster III included 2 wild tomato cultivars, LA1598 (35) and LA1777 (38), and Cluster IV included an individual cultivar, LA4096 (29).
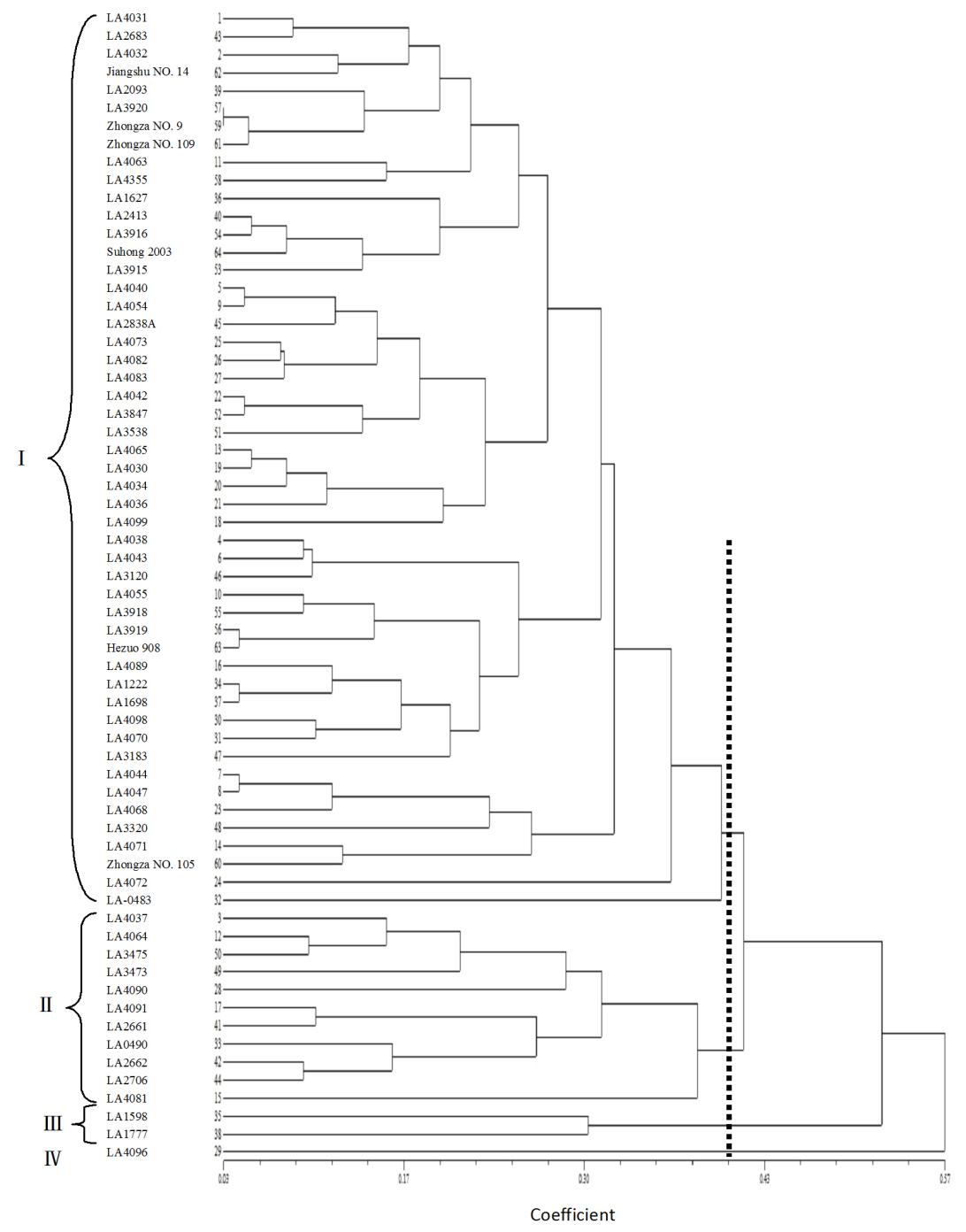

Figure 4. Dendrogram of 64 tomato cultivars based on RAPD marker data and generated from Nei's genetic distance matrix using UPGMA in NTSYSpc 2.11. 


\section{DISCUSSION}

The molecular characterization of cultivars is essential initial work for breeding, germplasm research, and seed sales in the commercial sector. In recent years, many molecular marker techniques based on DNA fingerprinting have been widely used in cultivar identification (Saker et al., 2006; Chiu et al., 2010) and genetic analyses (Boronnikova et al., 2007; Silvestrini et al., 2008; Bhau et al., 2009; Baysal et al., 2010). Because of the simple, rapid, and highly sensitive nature of the RAPD assay, it is fast becoming the most commonly used method for the molecular characterization of cultivars. In general, the application of RAPD markers in cultivar identification and genetic diversity detection includes the following procedure: first, a large number of primers are required for RAPD-PCR amplification and then electrophoresis; second, polymorphic fragments are scored using a binary matrix; third, genetic distance and clustering analysis with the aid of software are used to analyze genetic diversity; and finally, the results are output as a digital image. However, the identification process is masked in the sense that it is difficult to confirm which primer can be used to distinguish the target cultivar from others. Based on diversity analysis among given cultivars, when new cultivars are added, all primers used in the previous analysis need to be applied to the new cultivar for amplification, and these new data will be used to supplement the original work. In addition, the diversity analysis among all cultivars will be recalculated.

Although RAPD markers have been used for diversity analysis, they are not well managed. CID is a novel strategy for cultivar identification that transforms DNA fingerprinting into diagram information that is marked during the identification process. Therefore, this technique is regarded as one of the most intuitive forms of DNA fingerprinting application. As previously mentioned, CIDs have been successfully used in several fruit crop cultivars, such as sweet orange, pear, grapevine, loquat, and apple (Lin et al., 2011; Zhang et al., 2011; Zhao et al., 2011; Sun et al., 2012; Wang et al., 2012). In this study, the CID approach was first adopted to identify tomato cultivars, and then the entire appraisal process was recorded by CID, which provides information pertaining to the polymorphic primer and specific bands that were used for identification (Figure 2).

As opposed to reported molecular marker-based characterization for cultivar classification, the CID method overcomes the previously described deficiencies. For instance, fewer primers are used in the CID approach. In this paper, only 8 primers were needed to completely separate 64 tomato cultivars, using less time and work than other methods. Also, the CID exhibited better repeatability and reliability. In addition, the most obvious advantage in favor of the CID method is that the evaluation process shows detailed identification information in a diagrammatic format. From the CID of the 64 tomato cultivars, we can easily identify the exact primers that were used to distinguish the target cultivar from others. Furthermore, any randomly selected cultivars from the original 64 cultivars could be distinguished directly by the primers reported in the CID. For example, LA4072 is first separated from other tomato cultivars using primer Y42 in the CID; therefore, to distinguish LA4072, the Y42 primer is sufficient. However, according to genetic distance and clustering analysis, all primers should be used to amplify the cultivars to be detected; then, they can be compared by clustering analysis for further identification. Another advantage of CID is that it is not limited to the existing cultivars; when new tomato cultivars need to be identified, the 8 primers will be initially considered for use. If the 8 primers can completely separate these variants, the process can be directly added into the original CID. If this cannot be done, more primers will be screened 
and used for further identification, and then the new data will be used to supplement the CID.

In the research described herein, 8 primers were utilized to amplify 64 tomato cultivars. According to the 8 primers and the specific bands that were subsequently generated, a tomato CID was constructed. In addition, genetic diversity analysis was done using the same RAPD profiles. After RAPD-PCR amplification and electrophoresis, polymorphic fragments were scored as either 0 or 1 , and genetic distance and clustering analysis were performed by software. In this study, genetic relationships among cultivars could be clearly clarified by clustering analysis; cultivars with a close genetic relationship fall into the same cluster, otherwise, they fall into different clusters. CIDs can also be used to identify genetic relationships. Usually, it is relatively easy to use CID to distinguish distant genetic relationships; in contrast, however, a close genetic relationship often requires more primers. In this study, clustering analysis (Figure 4) placed LA3920 and Zhongza No. 9 in the same cluster, which suggested that they shared a very close genetic relationship. In the CID (Figure 2), we found that 7 RAPD primers were needed to separate LA3920 from Zhongza No. 9, suggesting that these 2 cultivars shared a similar genetic background. Similarly, to distinguish between LA4065 and LA4030, we also needed to use 7 RAPD primers in the CID (Figure 2), and clustering analysis showed that LA4065 and LA4030 were in the same cluster (Figure 4). These data suggest that there is excellent agreement between the CID and clustering analysis.

CID is an effective strategy for cultivar identification with features including simplicity, speed, and reliability. However, it is not without certain limitations. For instance, when cultivars need to be identified from the constructed CID, we can utilize the specific primers in the CID to distinguish the cultivars with fewer steps. However, for new cultivars that are not included in the CID, existing primers will be used, but if this is not sufficient, more primers will be screened until all cultivars are completely separated. Although CID can be used to estimate the general genetic diversity among cultivars, it cannot provide precise, accurate analysis. In this study, CID could accurately identify different cultivars, but it only offered a general judgment of the genetic diversity among cultivars.

In conclusion, this study proposes a novel strategy using the same RAPD profiles to simultaneously construct a CID and clustering analysis of 64 tomato cultivars. Compared to previously used methods, this novel strategy appears more convenient, efficient, and rapid for systematically understanding cultivar category and genetic diversity among tomatoes.

\section{ACKNOWLEDGMENTS}

Research supported by grants from the Priority Academic Program Development of Jiangsu Higher Education Institutions (PAPD), the National Undergraduate Innovative Experimentation Program (NUIEP, \#111030723), and the Research Innovation Program for College Graduates of Jiangsu Province (\#CXZZ12-0285).

\section{REFERENCES}

Aruga D, Tsuchiya N, Matsumura H, Matsumoto E, et al. (2012). Analysis of RAPD and AFLP markers linked to resistance to Fusarium oxysporum f. sp. lactucae race 2 in lettuce (Lactuca sativa L.). Euphytica 187: 1-9.

Baird WV, Ballard RE, Rajapakse S and Abbott AG (1996). Progress in Prunus mapping and application of molecular markers to germplasm improvement. HortScience 31: 1099-1106.

Baysal O, Siragusa M, Gümrükcü E, Zengin S, et al. (2010). Molecular characterization of Fusarium oxysporum $\mathrm{f}$. melongenae by ISSR and RAPD markers on eggplant. Biochem. Genet. 48: 524-537. 
Bhau BS, Medhi K, Das AP, Saikia SP, et al. (2009). Analysis of genetic diversity of Persea bombycina "Som" using RAPD-based molecular markers. Biochem. Genet. 47: 486-497.

Boronnikova SV, Kokaeva ZG, Gostimskii SA, Dribnokhodova OP, et al. (2007). Analysis of DNA polymorphism in a relict Uralian species, yellow foxglove (Digitalis grandiflora Mill.), using RAPD and ISSR markers. Genetika 43: 653-659.

Bousquet J, Simon L and Lalonde M (1990). DNA amplification from vegetative and sexual tissues of trees using polymerase chain reaction. Can. J. Forest Res. 20: 254-257.

Chiu TH, Pang JC, Chen MH and Tsen HY (2010). Improvement of strain discrimination by combination of RAPD with PFGE for the analysis of the swine isolates of Salmonella enterica serovar Choleraesuis. World. J. Microbiol. Biotechnol. 27: 465-469.

Comlekcioglu N, Simsek O, Boncuk M and Aka-Kacar Y (2010). Genetic characterization of heat tolerant tomato (Solanum lycopersicon) genotypes by SRAP and RAPD markers. Genet. Mol. Res. 9: 2263-2274.

Fang J, Twito T, Zhang Z and Chao CT (2006). Genetic relationships among fruiting-mei (Prunus mume Sieb. et Zucc.) cultivars evaluated with AFLP and SNP markers. Genome 49: 1256-1264.

Lee GP, Lee CH and Kim CS (2004). Molecular markers derived from RAPD, SCAR, and the conserved 18S rDNA sequences for classification and identification in Pyrus pyrifolia and P. communis. Theor. Appl. Genet. 108: 1487-1491.

Lin J, Wang XC, Chang YH and Fang JG (2011). Development of a novel and efficient strategy for practical identification of Pyrus spp (Rosaceae) cultivars using RAPD fingerprints. Genet. Mol. Res. 10: 932-942.

Murray GC and Thompson WF (1980). Rapid isolation of high molecular weight DNA. Nucleic Acids Res. 8: 4321-4325.

Palombi MA and Damiano C (2002). Comparison between RAPDs and SSR molecular markers in detecting genetic variation in kiwifruit (Actinidia deliciosa A. Chev). Plant Cell Rep. 20: 1061-1066.

Peralta IE, Knapp S and Spooner DM (2006). Nomenclature for wild and cultivated tomatoes. TGC Report 56: 6-12.

Rick CM, Laterrot H and Philouze J (1990). A revised key for the Lycopersicon species. TGC Report 40: 31.

Rohlf F (2000). NTSYS-PC numerical taxonomy and multivariate analysis system ver 2.11L. Applied Biostatistics, New York.

Saker MM, Adawy SS, Mohamed AA and El-Itriby HA (2006). Monitoring of cultivar identity in tissue culture-derived date palms using RAPD and AFLP analysis. Biol. Plantarum 50: 198-204.

Silvestrini M, Maluf MP, Silvarolla MB, Guerreiro-Filho O, et al. (2008). Genetic diversity of a Coffea germplasm collection assessed by RAPD markers. Genet. Resour. Crop Evol. 55: 901-910.

Sun X, Mu Q, Jiang D, Wang C, et al. (2012). A new strategy employed for identification of sweet orange cultivars with RAPD markers. Genet. Mol. Res. 11: 2071-2080.

Wang WY, Wang K, Liu FZ and Fang JG (2012). An efficient identification of 68 apple cultivars using a cultivar identification diagram (CID) strategy and RAPD markers. Korean J. Hortic. Sci. 30: 549-556.

Williams JGK, Kubelik AE, Livak KJ, Rafalski JA, et al. (1990). DNA polymorphisms amplified by arbitrary primers are useful as genetic markers. Nucleic Acids Res. 18: 6531-6535.

Yu HP, Fang JG, Zhang MY, Yang G, et al. (2009). Study on application of RAPD marker in cultivar identification of seven fruit crops. Acta Agric. Jiangxi 21: 5-9.

Zhang XY, Qian JL, Wang HK, Yuan WM, et al. (2011). A novel strategy employed in identification of 25 important loquat cultivars using RAPD marker. Caryologia 64: 265-271.

Zhao MZ, Zhang YP, Wu WM, Wang C, et al. (2011). A new strategy for complete identification of 69 grapevine cultivars using random amplified polymorphic DNA (RAPD) markers. Afr. J. Plant Sci. 5: 273-280. 